\title{
Cor Triatriatum Sinistrum: A Rarest Aetiology of Cardiac Chamber Thrombi Mimicking Left Atrial Mass
}

\author{
Osca Imatsu ${ }^{1}$, Budi Susetyo Pikir ${ }^{2}$, Ricardo Adrian Nugraha ${ }^{1 *}$ \\ ${ }^{1}$ Department of Cardiology and Vascular Medicine, Faculty of Medicine, Universitas \\ Airlangga, Surabaya \\ ${ }^{2}$ Division of Cardiovascular Imaging, Department of Cardiology and Vascular Medicine, \\ Faculty of Medicine, Universitas Airlangga - Dr. Soetomo General Hospital, Surabaya \\ * Corresponding author: Ricardo Adrian Nugraha, M.D. \\ Corresponding email: ricardo.adrian.nugraha-2019@fk.unair.ac.id
}

\begin{abstract}
Background. Cor triatriatum is a rare congenital cardiac anomaly, represent $0.1 \%$ of all congenital cardiac malformations and may be associated with other cardiac diseases in as many as $50 \%$ of cases. The natural history of this defect depends on the size of the communicating orifice between the upper and lower atrial chamber.

Case Presentation. We reported case of cor triatriatum in a 12 years old girl with chief complaint of shortness of breath, middle chest discomfort and palpitation since 5 days prior admission. The diagnosis was based on clinical features, chest radiography, electrocardiography and transthoracic echocardiography. Chest radiograph showed rounded cardiac apex and double contour appearance. ECG showed sinus rhythm, 75 beat per minute, RAD, CCWR, RVH, RV strain pattern with ST depression and T-wave inversion in II, III, aVF, $V_{1}-V_{5}$. TTE revealed 2 chambers of left atrium, with restrictive supramitral membrane, dilated right atrium, right ventricle and left atrium, smallish left ventricle, proximal left atrial thrombus $(5.96 \times 3.44 \mathrm{~cm})$, relative mitral stenosis, severe mitral regurgitation, mild aortic regurgitation and severe tricuspid regurgitation. A diagnosis of cor triatriatum sinister was made. The only treatment is surgical correction. Medical therapy, with ampicillin and heparin, was administered during admission.

Conclusion. Cor triatriatum has been reported in a 12-year-old girl. The diagnosis is confirmed by clinical manifestations, chest radiography and echocardiography. The only therapy is surgical correction. From the field of cardiac surgeon, patients are advised to improve their general conditions before underwent surgical procedures.
\end{abstract}

Keywords : Cor triatriatum, children, restrictive supramitral membrane, thrombus 


\section{Introduction}

Cor triatriatum is a very rare inherited disorder. In this disorder, the fibrous connective tissue will divide the right or left atrium into 2 parts [1-2]. The most common form, about $54 \%$ is the cor triatriatum sinister [3], the left atrium is divided into an upper chamber that receives the pulmonary veins and a lower chamber that is associated with the left atrial appendage (LAA) and mitral valve orifice. However, a variable type of subtotal cor triatriatum was also noted, with only the right or left pulmonary veins draining into the upper chambers [4]. The incidence is only $0.1 \%$ of all congenital heart defects and is usually found together with other heart defects in $50 \%$ of cases [5-6].

The clinical course depends on the relationship between the upper and lower chambers of the divided atrium [7]. When the connecting opening is small, the patient is critically ill, presenting from birth and usually dies at a young age (in infancy) due to congestive heart failure and pulmonary oedema. If the connector is large, complaints will appear in children or adults with clinical symptoms resembling mitral stenosis. Approximately $75 \%$ of patients die in infancy (usually from pulmonary hypertension) if the defect is not repaired [7]. If the relationship between the proximal and distal chambers is not restrictive or if the atrial septal defect can suppress left atrial hypertension, the prognosis is significantly improved [7, 9-10].

The only therapy is surgical correction. Most common postoperative mortality occur within the first 30 days. The early mortality rate in each major case series is consistent between $15-20 \%$. Early death has a higher rate of associated severe heart defects [3]. The long-term outcome is excellent, with long-term survival of $80-90 \%$ in patients who survive surgery [6].

The purpose of this case report is to make us more aware of this disease, knowing its clinical symptoms, diagnosis, treatment, and prognosis. 


\section{Case Illustration}

A 12 year old girl was referred from rural hospital at Sidoarjo town to our hospital on March 27, 2021 due to congenital heart disease. The patient came with complaints of shortness of breath since 5 days, breathless, unable to walk far, sometimes chest pain and palpitations. Currently the shortness of breath has decreased, sometimes coughing, intermittent chest pain, no fever and lack of appetite.

The patient has been regularly attending the congenital cardiac outpatient centre since 2 years ago, taking 3 kinds of drugs (the medicine was not brought and the mother forgot her name). History of febrile seizure last 2 years. The patient is the third child, born normal at term, the mother does not remember birth weight (BBL), not blue and not yellow. The patient did not receive immunizations. History of breastfeeding and formula feeding aged 0-8 months, complementary foods for breastfeeding since the age of 7 months. The patient has a history of delayed growth and development, namely walking at the age of 2.5 years and can only speak at the age of 2 years.

Upon admission to the hospital (March 27, 2021) based on a physical examination, the patient appeared short of breath and pale. The respiratory rate is $28 \mathrm{x} /$ minute with blood pressure $80 / 50$ $\mathrm{mmHg}$ and heart rate is $105 \mathrm{x} /$ minute regularly. Armpit temperature was $36.8 \mathrm{C}$. The patient's body weight is $19 \mathrm{~kg}$ compared to the ideal body weight, so the nutritional status of the patient is classified as moderate malnutrition. Oxygen saturation was $98 \%$ in room air.

The patient's conjunctiva was anaemic, the sclera was not icteric and the lips were not cyanotic. No enlarged lymph nodes or neck stiffness. On chest examination, a bulging chest was found. There were no subcostal, intercostal or suprasternal retractions. Breath sounds are vesicular, without crackles or wheezing. The ictus cordis can be palpated at the left midclavicular line V ICS. Thrill not palpable. Auscultation revealed a systolic murmur and a diastolic murmur at the heart apex of grade III/VI. Abdominal examination revealed a sob with normal bowel sounds. The liver and spleen are not palpable. Capillary refill time is less than 2 seconds and there is no oedema in the extremities.

The results of laboratory tests showed WBC 10,860 cells / uL, Hb 10.8 g/dL, HCT 33.9\%, PLT 230,000 cells /uL, CRP $3.2 \mathrm{mg} / \mathrm{L}$, blood sugar $97 \mathrm{mg} / \mathrm{dL}$, sodium $134 \mathrm{mmol} / \mathrm{L}$, potassium 3.2 mmol/L, chloride $92 \mathrm{mmol} / \mathrm{L}$, calcium $7.9 \mathrm{mmol} / \mathrm{L}$, PPT 13.1 seconds, APTT 24.9 seconds, SGOT $17 \mathrm{U} / \mathrm{L}$, SGPT $10 \mathrm{U} / \mathrm{L}, \mathrm{BUN} 29 \mathrm{mg} / \mathrm{dL}$, SK $0.7 \mathrm{U} / \mathrm{L}$ and albumin $3.5 \mathrm{~g} / \mathrm{dL}$.

Electrocardiographic investigations showed sinus rhythm, 75 beats per minute, right axis deviation (RAD), counter clockwise rotation (CCWR), right ventricular hypertrophy (RVH), RV strain pattern with ST segment depression and T wave inversion in II, III, aVF, V1-V5. Chest X-ray showed cardiomegaly with cardiothoracic ratio (CTR) 67\%, heart apex rounded, waist bulging heart, splaying carina and double contour. There was also an increase in pulmonary vascular markings as a pattern of acute pulmonary edema (Figure 1). 
Echocardiography shows 2 left atrial (LA) chambers, with a restrictive supramitral membrane, dilated right atrium (RA), right ventricle (RV) and left atrium (LA), small left ventricle (LV), proximal left atrium thrombus measuring $5.96 \times 3.44 \mathrm{~cm}$ (Figure 2), relative mitral stenosis (MS) (Figure 3), severe mitral regurgitation (MR), mild aortic regurgitation (AR) and severe tricuspid regurgitation (TR) (Figure 4). Left ventricular systolic function was normal with an ejection fraction $(\mathrm{EF})$ of $64.41 \%$.

Based of clinical manifestations, electrocardiographic, radiological, and echocardiographic examinations, the final diagnosis was cor triatriatum sinister, thrombus in proximal LA, relative mitral stenosis, severe mitral regurgitation, mild aortic regurgitation, severe tricuspid regurgitation, and moderate malnutrition. The patient was treated in the Bona 2 Cardio room and treated with oxygen using nasal 3 liters per minute, maximum fluid input of 1,160 ml/24 hours, IV fluid $\mathrm{NaCl} 0.9 \% 500 \mathrm{ml} / 24$ hours, Heparin pump 15 units/kg/hour, injection of Ampicillin 4x500 mg Intravenous, Omeprazole 1x20 mg intravenously, Furosemide 3x10 mg orally, Spironolactone 1x20 mg orally, Lisinopril 1x2 mg orally, N-Acetyl Cysteine 3x200 mg orally, Sildenafil 4x6 mg orally and Paracetamol 4x250 mg orally as needed.

For further treatment, the patient will undergo surgery, but previously it was submitted in a heart conference. IEC has been carried out on the patient and family regarding the risks if surgery is not performed and if surgery is performed, the patient and family decide to agree. The results of the heart team discussion planned supramitral membrane resection and thrombus evacuation. From the field of TKV, patients are advised to improve their general and polyclinic conditions, when surgery will be carried out, the patient can be called back.

\section{Discussion}

The cor triatriatum sinister is the more common form than the cor triatriatum dextrum [3]. In the cor triatriatum sinister, the left atrium divides into an upper chamber that receives the pulmonary veins and a lower chamber that communicates with the left atrial appendage (LAA) and the mitral valve orifice [1-2]. The incidence is only $0.1 \%$ of all congenital heart defects and is usually found together with other heart defects in 50\% of cases. Examples of associated heart defects include atrial septal defect (ASD), persistent left superior vena cava (PLSVC) with unroofed coronary sinus, partial anomalous pulmonary venous connection (PAPVC), ventricular septal defect (VSD), and more complex cardiac lesions, such as tetralogy of Fallot (ToF), atrioventricular canal, and double outlet right ventricle (DORV) [3]. Based on the presence and localization of ASD, there are four types of cast triatriatum (Figure 5). Type 1 (interatrial septum intact), type 2 (ASD connected to the upper chambers of the atria), type 3 (ASD connected to the lower chambers of the atria), and type 4 (ASD connected to both chambers). In this case, the diagnosis was cor triatriatum sinister. An intact interatrial septum was found, which was anatomically similar to type 1 .

The clinical course depends on the relationship between the upper and lower chambers of the divided atrium [7].When the connecting opening is small, the patient is critically ill, presenting from birth and usually dies at a young age (in infancy) due to congestive heart failure and 
pulmonary oedema. If the connector is large, complaints will appear in children or adults with clinical symptoms resembling mitral stenosis. Cor triatriatum may also be an incidental finding if it is not obstructive. In this case, symptoms appeared at the age of 10 years, with congestive heart failure and pulmonary oedema due to complete obstruction of pulmonary venous outflow to the left heart, meaning that there was functionally no connecting hole between the upper and lower chambers of the atrium. That is, there may be a restrictive relationship but the flow is not visible on colour Doppler echocardiography.

Approximately $75 \%$ of patients die in infancy (usually from pulmonary hypertension) if the defect is not corrected [7]. If the relationship between the proximal and distal chambers is not limited or if the ASD can suppress left atrial hypertension, the prognosis is significantly improved [7, 9-10]. In these cases In this case, the patient survived the cor triatriatum with a restrictive supramitral membrane and underwent a 23-day hospital stay.

Cor triatriatum is basically a form of obstruction of the inflow to the left atrium and presents with signs and symptoms of pulmonary venous obstruction. Most patients present during infancy with a restrictive opening in the membrane. These infants usually present with signs of low cardiac output, including pallor, a small peripheral pulse, and tachypnoea and tachycardia. Difficulty eating, poor weight gain, and respiratory distress are common. The later presentation is a bit classic. However, when the patient is symptomatic, signs of pulmonary venous obstruction predominate. In these patients, the membrane may become calcified with the orifice becoming smaller or the patient may develop mitral valve insufficiency. These patients may also present with arrhythmias secondary to hypertension from enlarged atria. In this case, the patient was referred due to shortness of breath. The patient came with complaints of shortness of breath since 5 days, breathless, unable to walk far, sometimes chest pain and palpitations. Currently the shortness of breath has reduced, sometimes coughing, intermittent chest pain, no fever and lack of appetite.

The patient has been regularly attending the PPJT pediatric cardiac polyclinic for 2 years ago, taking 3 kinds of drugs (the medicine was not brought and the mother forgot her name). History of febrile seizure last 2 years. The patient is the third child, born normal at term, the mother does not remember birth weight (BBL), not blue and not yellow. The patient did not receive immunizations. History of breastfeeding and formula feeding aged 0-8 months, complementary foods for breastfeeding since the age of 7 months. The patient has a history of delayed growth and development, namely walking at the age of 2.5 years and can only speak at the age of 2 years.

Although there is no intracardiac defect, there is no characteristic murmur or pathognomonic physical feature. There are signs of pulmonary venous obstruction and pulmonary hypertension. Right ventricular elevation and a loud second pulmonary sound are common and may be accompanied by an early diastolic murmur due to pulmonary insufficiency. Crackles may be found at the lung bases. A soft continuous murmur may occur as it flows across the membrane. A murmur at the left sternal border is heard in a patient with ASD and a left-toright shunt. The rumbling diastolic murmur of MS at the apex is generally not heard in the 
triatriatum. In this case, there were no subcostal, intercostal or suprasternal retractions. Breath sounds are vesicular, crackles are heard, without wheezing. The ictus cordis can be palpated at the left midclavicular line V ICS. Thrill not palpable. Auscultation revealed a systolic murmur and a diastolic murmur at the heart apex of grade III/VI.

Other clinical manifestations are caused by low cardiac output such as: pallor, tachypnea, tachycardia, and small peripheral pulses. Children with cast triatriatum are usually small, suffer from poor weight gain. The patient may have dyspnea with a history of frequent pulmonary infections. They may have signs of right-sided heart failure, including enlarged peripheral veins, elevated jugular venous pressure (JVP), and hepatomegaly. Signs and symptoms of pulmonary hypertension can be severe. In this case, the patient showed tachypnea with a respiratory rate of $28 \mathrm{x} / \mathrm{min}$, good peripheral oxygen saturation, $98 \%$ on room air. The patient's body weight is $19 \mathrm{~kg}$ compared to the ideal body weight, so the nutritional status of the patient is classified as moderate malnutrition. The liver and spleen are not palpable. Capillary refill time is less than 2 seconds and there is no edema in the extremities.

From a chest radiograph, findings are usually nonspecific but may include pulmonary congestion with a pattern of acute pulmonary edema in the hilar region. The patient may have mild RV enlargement and a prominent pulmonary artery segment. Dilated proximal chambers can cause left atrial enlargement to appear. The presence of an associated ASD or PAPVC adds to the excess pulmonary circulation in pulmonary vein obstruction. Radiographs may also show significant right ventricular enlargement. In this case, chest X-ray showed cardiomegaly with CTR 67\%, heart apex rounded, waist bulging heart, splaying carina and double contour. There is also an increase in pulmonary vascular markings as a pattern of acute pulmonary edema.

Echocardiography is often sufficient for diagnosis and is the diagnostic modality of choice. The display of the membrane with two-dimensional imaging, evaluation of turbulence flow on color Doppler and determination of each gradient across the membrane on continuous wave Doppler can be done by echocardiography. Concomitant congenital abnormalities can also be described well by echocardiography. In this case, we found a membrane dividing the left atrium as a feature of the left cor triatriatum. Echocardiography also showed an intact inter-trial septum, thrombus in the proximal left atrium measuring $5.96 \times 3.44 \mathrm{~cm}$, relative MS, severe MR, mild AR and severe TR as concomitant cardiac abnormalities in the cor triatriatum.

The only therapy is surgical correction. Open correction is currently preferred over closed (percutaneous) procedures. This procedure is performed by cardiopulmonary bypass through an atrial incision with complete diaphragmatic resection. The goal of medical therapy in the cor triatriatum is to reduce symptoms caused by pulmonary venous congestion until definitive surgical therapy can be instituted. This therapy is continued in the postoperative period until pulmonary resistance decreases and right ventricular performance improves. The mainstay of therapy is inotropic agents and diuretics. In this case, the patient was treated with oxygen using nasal 3 liters per minute, maximum fluid input of 1,160 ml/24 hours, IVFD $\mathrm{NaCl} 0.9 \% 500$ $\mathrm{ml} / 24$ hours, Dobutamine pump $10 \mathrm{mcg} / \mathrm{kgBW} /$ hour, Heparin pump 15 units/kgBW/ hours, Ampicillin 4x500 mg intravenously, Omeprazole 1x20 mg intravenously, Furosemide 3x10 
mg orally, Spironolactone 1x20 mg orally, Lisinopril 1x2 mg orally, N-Acetyl Cysteine 3x200 $\mathrm{mg}$ orally, Sildenafil 4x6 mg orally and Paracetamol 4x250 mg orally if the temperature is > $37.5 \square$ C. And the patient was planned to be referred to a cardiovascular thoracic surgeon for an intra-atrial septectomy procedure. From the field of TKV, patients are advised to improve their general and polyclinic conditions, when surgery will be carried out, the patient can be called back.

The prognosis is poor in symptomatic infants without surgical repair with a mortality rate of $75 \%$. The presence of associated cardiac abnormalities adversely affects the prognosis. If the relationship between the proximal and distal chambers is not restrictive or if an ASD is present, the prognosis is improved. In this case, the patient survived the cor triatriatum with a restrictive supramitral membrane and underwent a 23-day hospital stay.

\section{Conclusion}

A case of cor triatriatum has been reported in a 12-year-old girl. The diagnosis is confirmed by clinical manifestations, chest radiography and echocardiography. The only therapy is surgical correction. From the field of cardiac surgeon, patients are advised to improve their general conditions before underwent surgical procedures.

\section{Acknowledgement}

We would like to thank Agus Subagjo and Achmad Lefi for their assistance to make proper view in echocardiography.

\section{Financial Support}

This research received no specific grant from any funding agency, commercial or not-for-profit sectors.

\section{Conflict of interest}

None.

\section{Ethical Standard}

None.

\section{Department and Institution where work was done}

The work was done in the Department of Cardiology and Vascular Medicine Dr. Soetomo General Hospital, Surabaya, Indonesia. 


\section{Notes on patient consent case report}

Written informed consent was obtained from parent(s).

\section{References}

1. Park MK. Pediatric Cardiology for Practitioners. Fourth edition. Philadelphia: Elsevier Inc; 2008.

2. Krazemen Z, Scheld H, Tjan TD, Kraseman T. Cor Triatriatum: Short Review of the Literaturs upon Ten New Case. Herz. 2007;32:506-10.

3. Alphonso N, Norgaard M, Newcomb A, Brizard C, Cochrane A. Cor Triatriatum: Presentation, Diagnosis and Long Term Surgical Result. Ann Thorac Surg. 2005;80:1666-71.

4. Horenstein MS. Cor Triatriatum (cited 2021 April 20). Available from URL: http://emedicine.medscape.com/article/919758-print.

5. Kazanci SY, Emanni S, McElhinney DB. Outcome After Repair of Cor Triatriatum. Am J Cardiol. 2011;09:1-5.

6. Lupinski WR, Shankar S, Wong KY, Chan YH, Vosloo S, Moll JJ. Cor Triatriatum: Clinical Presentation in 18 Cases. Asian Cardiovasc Thorac Ann. 2001;9:106-10.

7. Tahernia C, Aschraft KW, Tutuska PJ. The Diagnosis of Cor Triatriatum Sinistrum in Children: A Continuing Dilema. South Med J. 1999;92:218-22.

8. Thakrar A, Shapiro MD, Jassal DS, Neilan TG, King MEE, Abhara S. Cor Triatriatum: The Utility of cardiovascular imaging. Can J Cardiol. 2007;23(2):143-5.

9. Humpl T, Reinekker K, Manlhiot C, Dipchand A, Coles J, McCrinddle W. Cor Triatriatum sinistrum in childhood: A Single institution's experience. Can J Cardiol. 2010;26(7):371-6.

10. Lacis A, Zidere V, Lubaua I, Straume Z, Auziod J, Dmits L. Cor Triatriatum associated with complex hearth defect. Acta Medica Lituanica. 2004;11(4):59-63. 
Figure Legends.

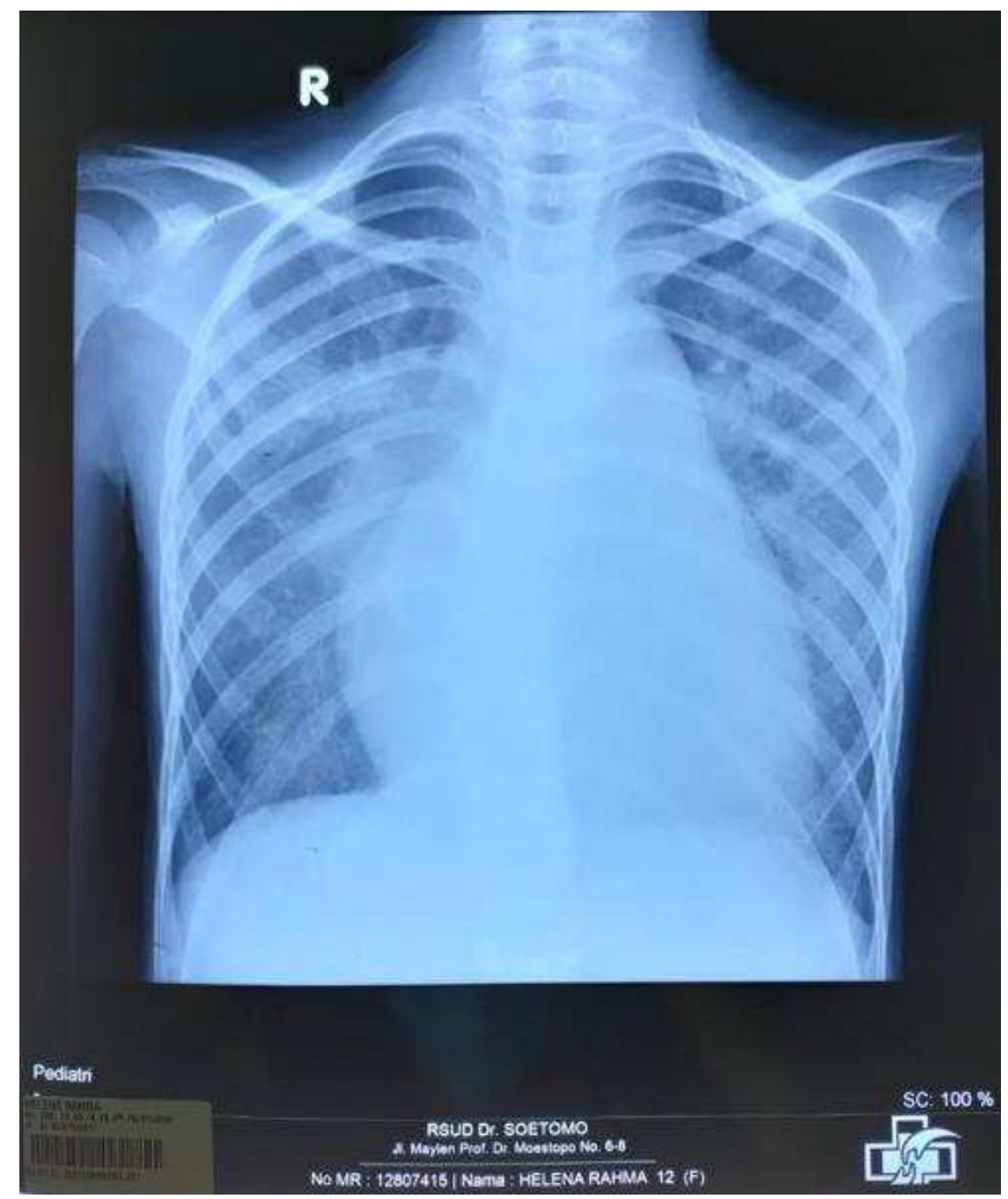

Figure 1. Chest X-ray examination showed marked enlargement of the heart 

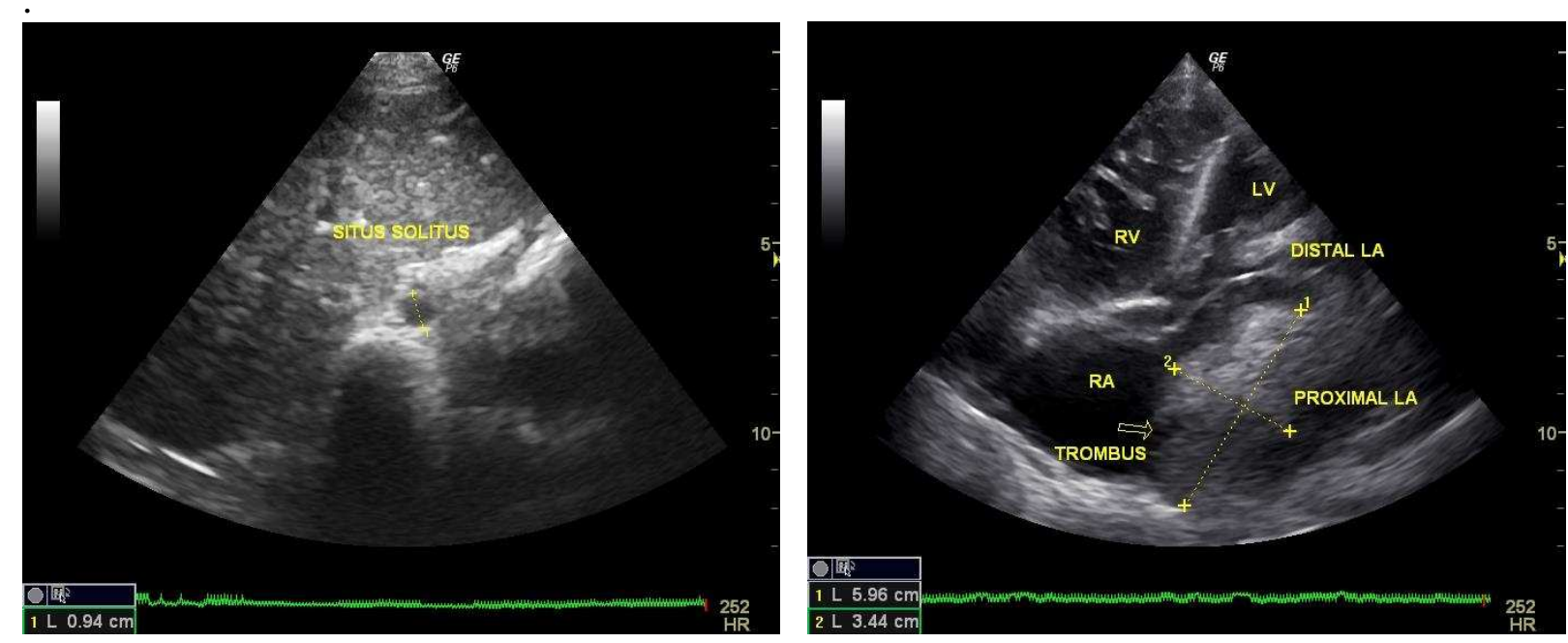

Figure 2. Left : TTE shows the solitus site. Right: TTE shows a restrictive supramitral membrane, dilated RA, RV and LA, small LV and thrombus proximal to LA $(5.96 \times 3.44 \mathrm{~cm})$. 

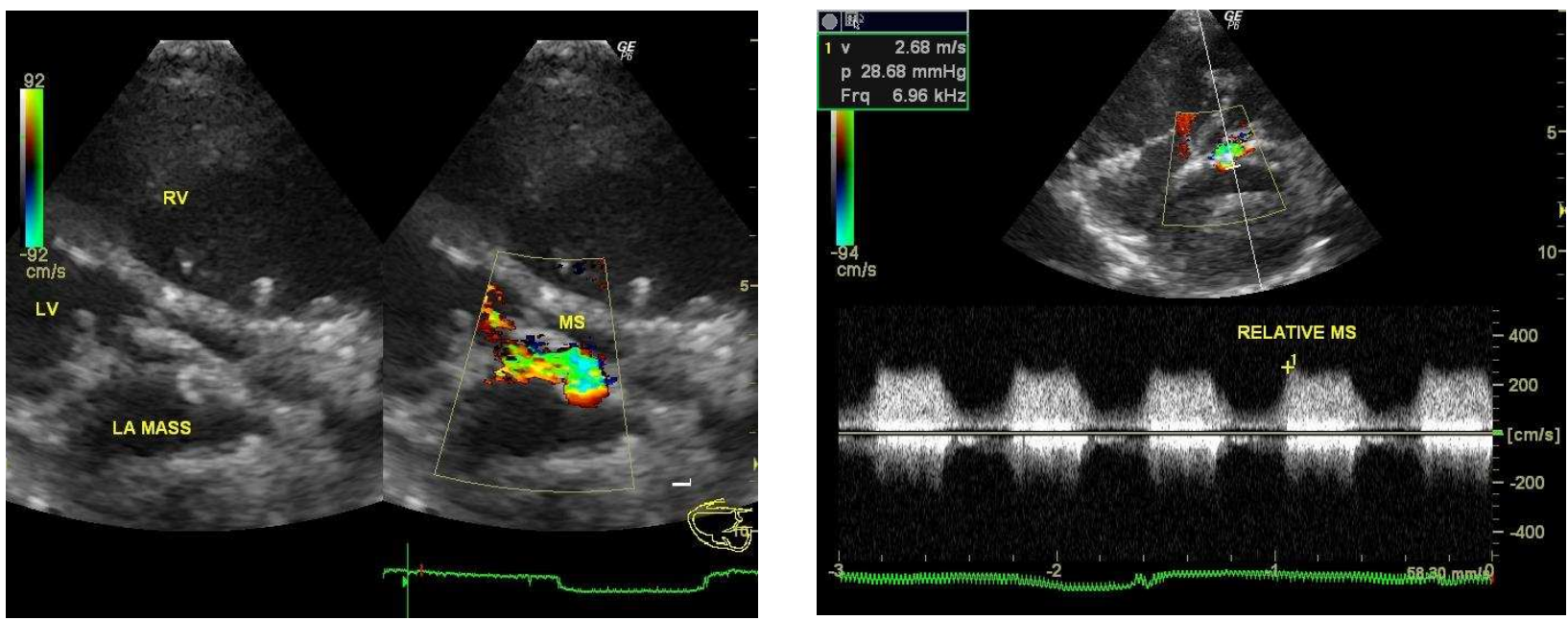

Figure 3. Transthoracic echocardiogram revealed relative mitral stenosis with peak gradient $28.68 \mathrm{mmHg}$
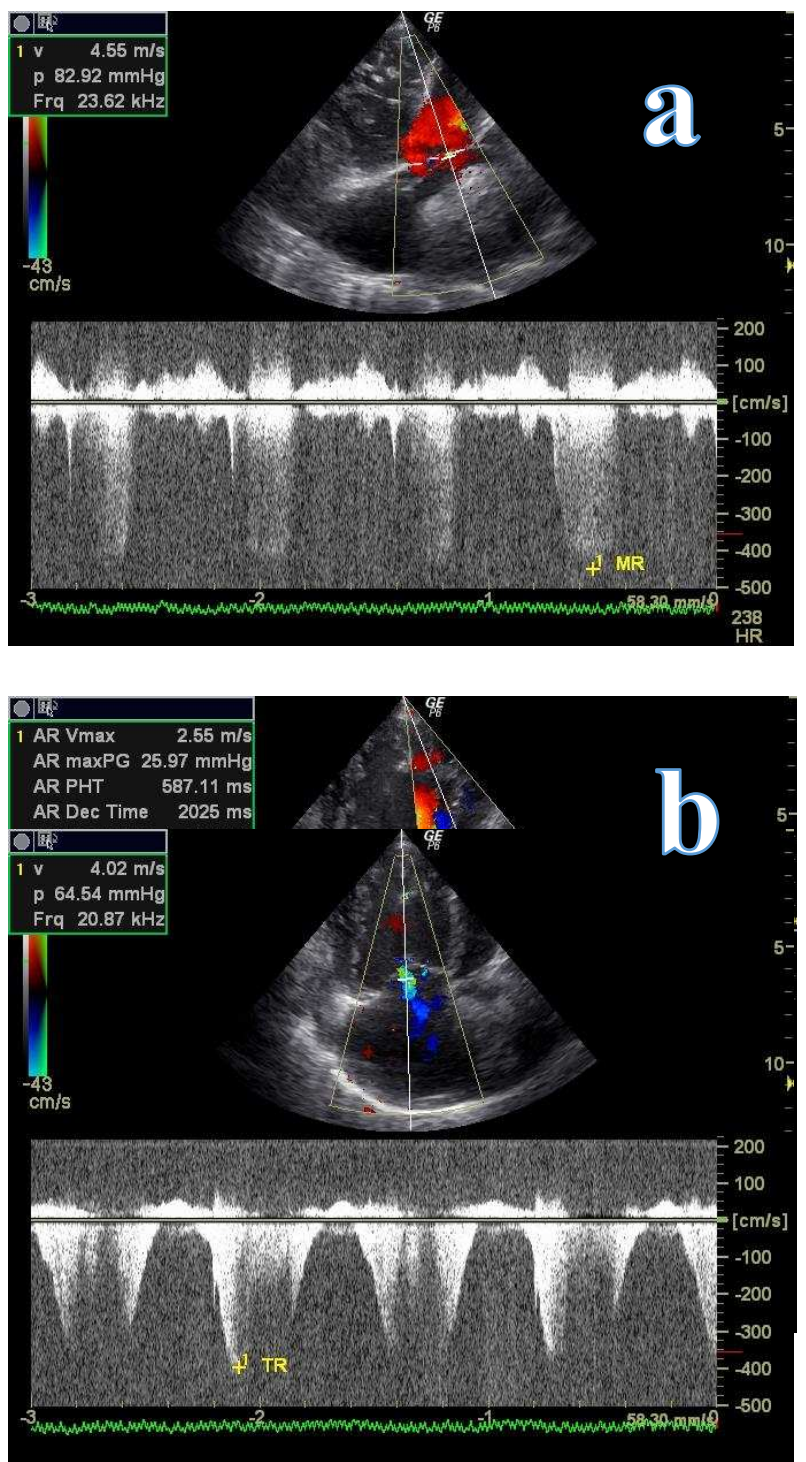
Figure 4. (a) Transthoracic echocardiogram showed severe mitral regurgitation (PG 82.92 $\mathrm{mmHg}$ ). (b) Transthoracic echocardiogram showed mild aortic regurgitation (SDec $1.26 \mathrm{~m} / \mathrm{s}^{2}$ ). (c) Transthoracic echocardiogram indicated severe tricuspid regurgitation (PG $64.54 \mathrm{mmHg}$ ).
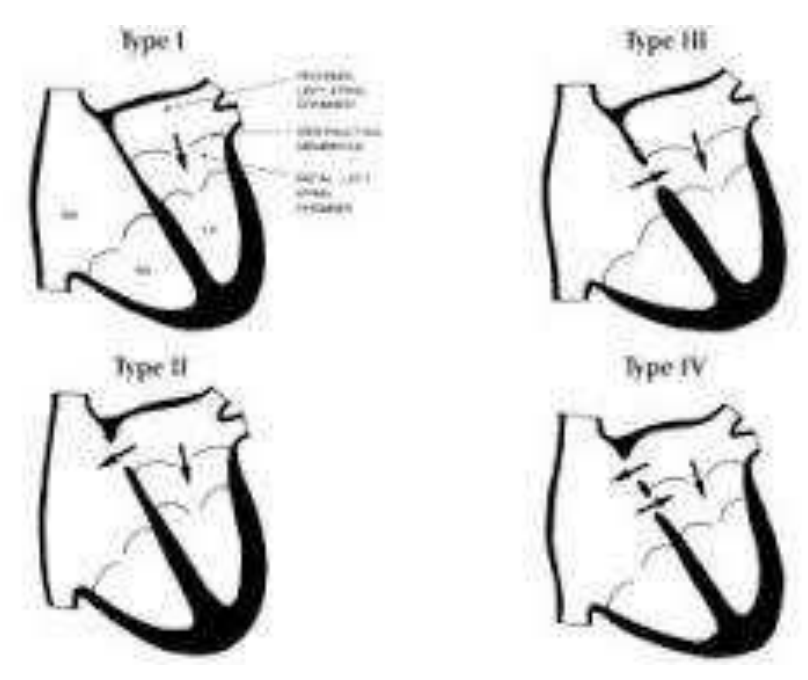

Figure 5. Anatomical type of cor triatriatum. 